\title{
THE MODEL FOR FINAL STAGE OF GRAVITATIONAL COLLAPSE MASSLESS SCALAR FIELD
}

\author{
V.D. Gladush ${ }^{1}$, D.V. Mironin ${ }^{2}$ \\ Dnipropetrovsk National University, Gagarin Ave, 725014, Ukraine, \\ 1vgladush@gmail.com,2dmyronin1993@mail.ru
}

\begin{abstract}
It is known that in General relativity, for some spherically symmetric initial conditions, the massless scalar field (SF) experience the gravitational collapse (Choptuik, 1989), and arise a black hole (BH). According Bekenstein, a BH has no "hair scalar", so the SF is completely under the horizon. Thus, the study of the final stage for the gravitational collapse of a SF is reduced to the construction of a solution of Einstein's equations describing the evolution of a SF inside the $\mathrm{BH}$. In this work, we build the Lagrangian for scalar and gravitational fields in the spherically symmetric case, when the metric coefficients and SF depends only on the time. In this case, it is convenient to use the methods of classical mechanics. Since the metric allows an arbitrary transformation of time, then the corresponding field variable $\left(g_{00}\right)$ is included in the Lagrangian without time derivative. It is a non-dynamic variable, and is included in the Lagrangian as a Lagrange multiplier. A variation of the action on this variable gives the constraint. It turns out that Hamiltonian is proportional to the constraint, and so it is zero. The corresponding Hamilton-Jacobi equation easily integrated. Hence, we find the relation between the SF and the metric. To restore of time dependence we using an equation $\partial L / \partial \dot{q}=\partial S / \partial q$ After using a gauge condition, it allows us to find solution. Thus, we find the evolution of the SF inside the $\mathrm{BH}$, which describes the final stage of the gravitational collapse of a SF. It turns out that the mass $\mathrm{BH}$ associated with a scalar charge $G$ of the corresponding SF inside the $\mathrm{BH}$ ratio $M=G /(2 \sqrt{ } \kappa)$.
\end{abstract}

Keywords: scalar field, black hole, Einstein equations.

\section{Introduction}

One of the most interesting objects in astrophysical and cosmological applications of General relativity (GR) is a scalar field. However, the models with scalar field known only for the simplest configurations, due to the difficulties of obtaining analytical solutions. So for a massless scalar field in General relativity is widely known spherically symmetric static solution of Fisher (1948) and its various later modifications (Janis, Newman, Winicour (1968); Wyman (1981); Agnese and La Camera. (1985)). Analytical solutions for the system of static scalar and electromagnetic fields in General relativity for spherical symmetry built Bronnikov (1972), Zaitsev, Kolesnikov and Radynov (1972), and then Korkina (1976) in different coordinate systems. All these solutions are unstable and have naked singularity. It turns out that for systems with a scalar field analogue of Birkhoff theorem on the uniqueness of the solution fails, and in addition, there is no conserved scalar charge. Therefore, a static configuration with a scalar field which satisfies the Einstein equations, due to negligible fluctuations, coming out of this mode and begins to evolve.

For dynamic spherically symmetric problems, due to the obvious difficulties in obtaining solutions of Einstein's equations in closed form, most of the work performed numerically. Roberts (1989) has built one of the few analytical solutions in closed form. Initially, he scheduled to use it as a counter-example to the hypothesis of cosmic censorship. However, later Brady (1994); Oshiro, Nakamura and Tomimatsu (1994) rediscovered this solution in the context of critical gravitational collapse

Spherically symmetric collapse of a scalar field is the perfect model for studying the dynamics of strong field in general relativity. Therefore, in this model used analytical and numerical methods. In particular, Christodoulou (1987) rigorously established global existence and uniqueness of solutions of the Einstein equations for a scalar field. He proved that the space of General relativity together with spherically symmetric scalar field with a sufficiently weak (in some sense) initial data evolves in Minkowski space-time, while the class sufficiently strong data forms a $\mathrm{BH}$.

The behavior of the scalar field near the threshold of $\mathrm{BH}$ formation was first investigated Choptuik (1993). He numerically solved the Einstein equations for spherically symmetric systems of the gravitational and massless scalar fields with minimal coupling. He studied the gravitational collapse for different sets of one-parameter families of the initial data. For example, they can be taken as a family of Gaussian pulses

$$
\varphi(0, v)=p v^{2} \exp \left(-\left(v-v_{0}\right)^{2} / \sigma^{2}\right) .
$$

Suppose that for a given family the parameter $p$ is chosen in such a way that for small values of $p$ the gravitational field in during the evolution of is too weak to form a $\mathrm{BH}$ (the field is scattered), while for large values of parameter $\mathrm{p}$ is formed $\mathrm{BH}$. Then between these two limits, there is a critical value of this parameter $p^{*}$ in which first formed the $\mathrm{BH}$. Solutions for which $p<p^{*}$ are called subcritical and solving for $p>p^{*}-$ supercritical, respectively. Choptuik proved that in the collapse may occur arbitrarily small $\mathrm{BH}$. Moreover, when $p>p^{*}$ the mass of the $\mathrm{BH}$ is given by 


$$
M_{B H}(p) \propto\left|p-p^{*}\right|^{\beta},
$$

where the exponent $\beta$ has a universal value $\gamma \simeq 0.374$ for all 1-parameter families of scalar field data. There are still a number of other features critical gravitational collapse of a scalar field, for example, discrete and continuous selfsimilarity, and others. Work Choptuik is an example of when the discovery of a new phenomenon in General relativity was done numerically. The discovery of universal properties of critical collapse is one of significant achievements the numerical relativity (Novikov \& Frolov, 2001).

\section{The action and its reduction}

Consider the evolution of spherically symmetric massless scalar field in the supercritical case, when $p>p^{*}$. In this case, at the final stage of the gravitational collapse is formed the black hole with a mass $M_{B H}(p)$. The residual relaxation phenomena associated with scattering of the remnants of the scalar field at infinity, do not affect the black hole and can be ignored.

According to the no-hair theorem Chase (1970) and Bekenstein (1972), the BH has no of neutral "scalar hairs" (as, indeed, and charged), therefore on the final stage of the gravitational collapse a scalar field is completely under the horizon, inside a black hole. Beyond the $\mathrm{BH}$, we have the free vacuum gravitational field described by the Schwarzschild solution. Thus, the study of the final stage the gravitational collapse a scalar field, by definition, is to construction of the solution of Einstein equations describing the evolution of scalar field inside the $\mathrm{BH}$ and satisfying appropriate boundary conditions.

In view with the above, we assume that the space-time inside a $\mathrm{BH}$ is described by the spherically symmetric metric, depending on time

$$
\begin{aligned}
d s^{2}= & e^{v(t)} c^{2} d t^{2}-e^{\lambda(t)} d r^{2}-e^{\mu(t)} d \sigma^{2}, \\
& d \sigma^{2}=d \theta^{2}+\sin ^{2} \theta d \alpha^{2} .
\end{aligned}
$$

For simplicity, consider the evolution of the homogeneous scalar field $\psi=\psi(\mathrm{t})$. Note that the metric admits an arbitrary gauge transformation time $t=t(\tilde{t})$, which induces the transformation of the metric coefficient $e^{\widetilde{v}}=e^{v}(d t / d \tilde{t})^{2}$. Thus, there is a gauge arbitrariness in the definition of the metric function $e^{v}=N^{2}$.

$$
\begin{gathered}
S=\int \Lambda \sqrt{-g} d^{4} x . \\
\Lambda=-\frac{1}{4 \pi}\left[\frac{c^{4}}{4 k} R-\frac{1}{2} g^{\mu \nu} \varphi,{ }_{\mu} \varphi,_{v}\right] .
\end{gathered}
$$

Here scalar curvature $\mathrm{R}$ in this case is equal to

$$
\begin{gathered}
R=-2 e^{-\mu}-\frac{e^{-v}}{c^{2}}\left(2 \ddot{\mu}+\ddot{\lambda}+\frac{3}{2} \dot{\mu}^{2}+\dot{\mu} \dot{\lambda}-\right. \\
\left.-\dot{\mu} \dot{v}+\frac{1}{2} \dot{\lambda}^{2}-\frac{1}{2} \dot{v} \dot{\lambda}\right), \\
\sqrt{-g}=e^{\mu+(v+\lambda) / 2} \sin \theta,
\end{gathered}
$$

where the point denotes $d / d x^{0}$. After the integration over the angles, the action takes the form

$$
\begin{gathered}
S=\int L d x^{0} d r \\
L=\frac{1}{2 c^{2}}\left[\frac{c^{4}}{2 k}\left(2 \ddot{\mu}+\ddot{\lambda}+\frac{3}{2} \dot{\mu}^{2}+\dot{\mu} \dot{\lambda}-\dot{\mu} \dot{v}+\frac{1}{2} \dot{\lambda}^{2}-\frac{1}{2} \dot{v} \dot{\lambda}\right)+\right. \\
\left.+\dot{\varphi}^{2}\right] e^{\frac{\lambda-v}{2}+\mu}+\frac{c^{4}}{2 k} e^{\frac{v+\lambda}{2}} .
\end{gathered}
$$

Since the Lagrangian and the field variables is independent from the coordinate $r$, it possible to be limited to the one-dimensional system with the action

$$
\widetilde{S}=\int L d x^{0}
$$

Extracting total time derivative, we obtain an effective Lagrangian

$$
\widetilde{L}=-\frac{1}{2 c^{2}}\left[\frac{c^{4}}{2 k}\left(\frac{1}{2} \dot{\mu}^{2}+\dot{\mu} \dot{\lambda}\right)-\dot{\varphi}^{2}\right] e^{\frac{\lambda-v}{2}+\mu}+\frac{c^{4}}{2 k} e^{\frac{v+\lambda}{2}} .
$$

For diagonalization of the Lagrangian, we make a change of field variables

Then

$$
\mu=\omega-\lambda, \quad v=\rho-\lambda .
$$

$$
\widetilde{L}=\frac{1}{2}\left[\frac{\dot{\varphi}^{2}}{c^{2}}-\frac{c^{3}}{4 k}\left(\dot{\omega}^{2}-\dot{\lambda}^{2}\right)\right] e^{\omega-\rho / 2}+\frac{c^{4}}{2 k} e^{\rho / 2} .
$$

In the new variables, the metric takes the form

$$
d s^{2}=e^{-\lambda}\left(e^{\rho} c^{2} d t^{2}-e^{\omega} d \sigma^{2}\right)-e^{\lambda} d r^{2} .
$$

\section{The equation of Hamilton-Jacobi in the} minisuperspace

To solve the problem under consideration is convenient to use the methods of classical mechanics. From the point of view of the classical mechanics, the metric coefficients and a scalar field in the resulting Lagrangian are the generalized coordinates (coordinates of a minisuperspace Wheeler-DeWitt).

Note that the metric allows arbitrary gauge transformation of time, and the corresponding metric variable $e^{\rho}$ enters the Lagrangian without time derivative. Therefore, it is a non-dynamic variable and is included in the Lagrangian as the Lagrange multiplier. Variation of the Lagrangian on this variable gives us the constraint:

$$
\frac{\partial \widetilde{L}}{\partial \rho}=-\frac{1}{4}\left[\frac{\dot{\varphi}^{2}}{c^{2}}-\frac{c^{2}}{4 k}\left(\dot{\omega}^{2}-\dot{\lambda}^{2}\right)\right] e^{\omega-\rho / 2}+\frac{c^{4}}{4 k} e^{\rho / 2}=0 .
$$

Next, we find momenta, conjugate of the dynamic variables $\{\varphi, \omega, \lambda\}$ :

$$
\begin{gathered}
p_{\varphi}=\frac{\partial \widetilde{L}}{\partial \dot{\varphi}}=\frac{\dot{\varphi}}{c^{2}} e^{\omega-\rho / 2}, \quad p_{\omega}=\frac{\partial \widetilde{L}}{\partial \dot{\omega}}=-\frac{c^{2}}{4 k} \dot{\omega} e^{\omega-\rho / 2}, \\
p_{\lambda}=\frac{\partial \widetilde{L}}{\partial \dot{\lambda}}=\frac{c^{2}}{4 k} \dot{\lambda} e^{\omega-\rho / 2},
\end{gathered}
$$

and Hamiltonian 
$H=\frac{1}{2}\left[\left(c^{2} p_{\varphi}{ }^{2}-\frac{4 k}{c^{2}}\left(p_{\omega}{ }^{2}-p_{\lambda}{ }^{2}\right)\right) e^{\frac{\rho}{2}-\omega}\right]-\frac{c^{4}}{2 k} e^{2}$.

Comparing the Hamiltonian with the constraint, it is easy to see that they are proportional

$$
H=-2 \frac{\partial \tilde{L}}{\partial \rho} .
$$

Hence, by virtue of Lagrangian constraint $\partial \mathrm{L} / \partial \rho=0$ it follow the Hamiltonian constraint

$$
\frac{1}{2} c^{2} p_{\varphi}{ }^{2}-\frac{2 k}{c^{2}}\left(p_{\omega}{ }^{2}-p_{\lambda}{ }^{2}\right) e^{\frac{\rho}{2}-\omega}-\frac{c^{4}}{2 k} e^{\frac{\rho}{2}}=0 .
$$

The latter is a consequence of the invariance of the theory with respect to gauge transformations $t=t(\tilde{t})$ Next, substituting momenta

$$
p_{\varphi}=\frac{\partial S}{\partial \varphi}, \quad p_{\lambda}=\frac{\partial S}{\partial \lambda}, \quad p_{\omega}=\frac{\partial S}{\partial \omega}
$$

in the Hamiltonian constraint, we come to the HamiltonJacobi equation (HJE)

$$
c^{2}\left(\frac{\partial S}{\partial \varphi}\right)^{2}-\frac{4 k}{c^{2}}\left(\frac{\partial S}{\partial \omega}\right)^{2}+\frac{4 k}{c^{2}}\left(\frac{\partial S}{\partial \lambda}\right)^{2}=\frac{c^{4}}{k} e^{\omega} .
$$

This equation is one-dimensional minisuperspace analogue of the Peres equation in the functional derivatives in a superspace. The variable $e^{\rho / 2}$ is not included in the GJE and is not related with the minisuperspace dynamics. Thus, the 3-geometry minisuperspace with coordinates $\varphi, e^{\lambda}, e^{\omega}$ is defined by diffeomorphic invariant way. It is easy to see that this equation is the GJE for the geodesic in minisuperspace in terms minisupermetric (in a potential space). Indeed, let us rewrite the action for the diagonalized Lagrangian

$$
\widetilde{S}=\int\left[\left(\frac{\dot{\varphi}^{2}}{2 c^{2}}-\frac{c^{3}}{8 k}\left(\dot{\omega}^{2}-\dot{\lambda}^{2}\right)\right) e^{\omega-\rho / 2}+\frac{c^{4}}{2 k} e^{\rho / 2}\right] d x^{0}
$$

Nondynamical variable can be eliminated. In order to do this we find the variable $e^{\rho / 2}$ from the constraint equation

$$
e^{\rho / 2}=\frac{\sqrt{k}}{c^{2}} \sqrt{\frac{\dot{\varphi}^{2}}{c^{2}}-\frac{c^{2}}{4 k}\left(\dot{\omega}^{2}-\dot{\lambda}^{2}\right)} e^{\omega / 2},
$$

and substitute into the action. The result is

$$
\widetilde{S}=\int \widetilde{L} d x=\frac{c^{2}}{\sqrt{k}} \int e e^{\omega / 2} \sqrt{\frac{\dot{\varphi}^{2}}{c^{2}}-\frac{c^{2}}{4 k}\left(\dot{\omega}^{2}-\dot{\lambda}^{2}\right)} d x^{0} .
$$

From here we have

$$
\tilde{S}=\frac{c^{2}}{\sqrt{k}} \int e^{\omega / 2} \sqrt{\frac{d \varphi^{2}}{c^{2}}-\frac{c^{2}}{4 k}\left(d \omega^{2}-d \lambda^{2}\right)} .
$$

It is easy to show that the equations resulting from the variational principle $\delta S=0$ together with the constraint are equivalent to the Einstein equations for the original metric. Taking the differential from the action and placed in the square, we obtain the interval in a minisuperspace

$$
\begin{aligned}
d \tilde{S} & =\frac{c^{2}}{\sqrt{k}} e^{\omega / 2} \sqrt{\frac{d \varphi^{2}}{c^{2}}-\frac{c^{2}}{4 k}\left(d \omega^{2}-d \lambda^{2}\right)}, \\
d \tilde{s}^{2} & =G_{a b} d q^{a} d q^{b}=\frac{c^{4}}{k} e^{\omega}\left[\frac{d \varphi^{2}}{c^{2}}-\frac{c^{2}}{4 k}\left(d \omega^{2}-d \lambda^{2}\right)\right] .
\end{aligned}
$$

\section{Solutions of the Hamilton-Jacobi equation}

We are searching the solution GJE in the form

$$
S=a \lambda+b \varphi+V(\omega) .
$$

Then the function $\mathrm{V}(\omega)$ is determined by the integral

$$
V(\omega)=\int \sqrt{A^{2}-e^{\omega} \varepsilon^{2}} d \omega,
$$

where

$$
A^{2}=a^{2}+\frac{c^{4}}{4 k} b^{2}, \quad \varepsilon^{2}=\frac{c^{6}}{4 k^{2}} .
$$

Thus, we find for the action

$$
S=a \lambda+b \varphi+\int \sqrt{A^{2}-e^{\omega} \varepsilon^{2}} d \omega .
$$

From the relations

$$
\frac{\partial S}{\partial a}=\ln N, \quad \frac{\partial S}{\partial b}=\varphi_{0},
$$

we find the connection between the scalar field $\varphi$ and metric functions $\lambda, e^{\omega}$ In order to restore the time dependence, we use the equation

$$
p_{\omega}=\frac{\partial L}{\partial \dot{\omega}}=\frac{\partial S}{\partial \omega} .
$$

As a result, we obtain

$$
-\frac{c^{2}}{4 k} e^{\omega-\rho / 2} \dot{\omega}=\sqrt{A^{2}-e^{\omega} \varepsilon^{2}} .
$$

Choosing the gauge condition

$$
e^{\rho / 2}=1
$$

we find

$$
\int \frac{e^{\omega} d \omega}{\sqrt{A^{2}-e^{\omega} \varepsilon}}=-\frac{4 k}{c^{2}} t .
$$

Hence we obtain the equation

Where

$$
e^{\omega}=B^{2}-c^{2} t^{2} \text {. }
$$

$$
B^{2}=\frac{k}{c^{2}}\left(\frac{4 k}{c^{4}} a^{2}+b^{2}\right) .
$$

Thus the metric takes the form

$$
d s^{2}=e^{-\lambda}\left(e^{\rho} c^{2} d t^{2}-\left(B^{2}-c^{2} t^{2}\right) d \sigma^{2}\right)-e^{\lambda} d r^{2} .
$$

Furthermore, we find the remaining variables $\varphi$ and $\lambda$, as function of time t. From the relations 
it follows that

$$
\frac{\partial S}{\partial a}=\ln N, \quad \frac{\partial S}{\partial b}=\varphi_{0},
$$

$$
\begin{gathered}
\lambda+a \int \frac{d \omega}{\sqrt{A^{2}-e^{\omega} \varepsilon^{2}}}=\ln N, \\
\varphi+\frac{c^{4} b}{4 k} \int \frac{d \omega}{\sqrt{A^{2}-e^{\omega} \varepsilon^{2}}}=\varphi_{0} .
\end{gathered}
$$

Taking into account

$$
\begin{gathered}
e^{\omega}=B^{2}-c^{2} t^{2}, \quad B^{2}=\frac{k}{c^{2}}\left(\frac{4 k}{c^{4}} a^{2}+b^{2}\right), \\
A^{2}=a^{2}+\frac{c^{4}}{4 k} b^{2},
\end{gathered}
$$

from here we find

$$
\begin{gathered}
e^{\lambda}=N\left|\frac{B+c t}{B-c t}\right|^{p}, \quad p=\frac{2 a k}{B c^{3}}, \\
\varphi=\varphi_{0}+\frac{c b}{2 B} \ln \left|\frac{B+c t}{B-c t}\right|,
\end{gathered}
$$

where

$$
B^{2}=\frac{1}{c^{6}}\left(4 k^{2} a^{2}+k b^{2} c^{4}\right), \quad \mathrm{N}=\text { const. }
$$

Thus, the metric and the scalar field inside the BH take the form

$$
\begin{aligned}
& d s^{2}=N^{-1}\left(\frac{B+c t}{B-c t}\right)^{-p}\left[c^{2} d t^{2}-\left(B^{2}-c^{2} t^{2}\right) d \sigma^{2}\right]- \\
& -N\left(\frac{B+c t}{B-c t}\right)^{p} d r^{2} . \\
& \varphi=\varphi_{0}+\frac{c b}{2 B} \ln \left|\frac{B+c t}{B-c t}\right|, \quad-B<c t<B .
\end{aligned}
$$

\section{Determination constants}

To find the constant, we can use the correspondence principle. When the scalar field vanishes, the resulting solution should be the same with the Schwarzschild solution in the T-region. Therefore, we put $b=0, \varphi_{0}=0$. Then

$$
\begin{gathered}
B=\frac{2 k a}{c^{3}}, \quad \mathrm{p}=1 \\
d s^{2}=N^{-1}\left[\frac{2 k a / c^{3}+c t}{2 k a / c^{3}-c t} c^{2} d t^{2}-\left(\frac{2 k a}{c^{3}}-c t\right)^{2} d \sigma^{2}\right]- \\
-N \frac{2 k a / c^{3}+c t}{2 k a / c^{3}-c t} d r^{2} .
\end{gathered}
$$

After replacing

we have

$$
\begin{aligned}
d s^{2} & =N^{-1}\left[\left(\frac{4 k a}{c^{4} T}-1\right)^{-1} c^{2} d T^{2}-c^{2} T^{2} d \sigma^{2}\right]- \\
& -N\left(\frac{4 k a}{c^{4} T}-1\right) d r^{2} .
\end{aligned}
$$

This metric coincides with the Schwarzschild solution

$$
d s^{2}=\left(1-\frac{2 k m}{c^{2} R}\right) c^{2} d t^{2}-\left(1-\frac{2 k m}{c^{2} R}\right)^{-1} d R^{2}-R^{2} d \sigma^{2}
$$

in the T-region, when we replace $\mathrm{cT} \rightarrow \mathrm{R}, \mathrm{r} \rightarrow \mathrm{ct}$ and choice the constants $\mathrm{N}=1, a=m c / 2$. Thus, the solution can be written in the form

$$
\begin{gathered}
d s^{2}=\left(\frac{B+c t}{B-c t}\right)^{-p}\left[c^{2} d t^{2}-\left(B^{2}-c^{2} t^{2}\right) d \sigma^{2}\right]- \\
-\left(\frac{B+c t}{B-c t}\right)^{p} d r^{2}, \\
\varphi=\varphi_{0}+\frac{G}{2 B} \ln \left|\frac{B+c t}{B-c t}\right|, \\
-B<c t<B, \quad B=\frac{1}{c^{2}} \sqrt{k^{2} m^{2}+k G^{2}}, \\
p=\frac{k m}{\sqrt{k^{2} m^{2}+k G^{2}}},
\end{gathered}
$$

where we introduced the scalar charge $G=c b$.

\section{Conclusion}

In the obtained solution about free scalar field, except the scalar charge, any other constants should not be. Therefore, we should put $\mathrm{m}=0$ and $\mathrm{p}=0$. Then we have

$$
B=\frac{\sqrt{k} G}{c^{2}} \text {. }
$$

In this case, the metric and scalar field take the form

$$
\begin{gathered}
d s^{2}=c^{2} d t^{2}-d r^{2}-c^{2}\left(t_{G}{ }^{2}-t^{2}\right) d \sigma^{2}, \\
\varphi=\frac{c}{2 \sqrt{k}} \ln \left|\frac{t_{G}+t}{t_{G}-t}\right|, \quad-t_{G}<t<t_{G} .
\end{gathered}
$$

where we have introduced the constant

$$
t_{G}=\frac{B}{c}=\frac{\sqrt{k} G}{c^{3}} .
$$

We take into account that at a maximum expansion (at the boundary of $\mathrm{BH}$ ), when $t=t_{G}$, the scalar field vanishes, $\varphi_{0}=0$. This metric correspond the scalar field confinement under the horizon $\mathrm{BH}$ by the gravitational interaction.

Note that the spatial part of the metric describes a metric of hypercylinder $R^{1} \otimes S^{2}$. 


$$
d l^{2}=d r^{2}+c^{2}\left(t_{G}{ }^{2}-t^{2}\right) d \sigma^{2} .
$$

Here $R=c \sqrt{t_{G}{ }^{2}-t^{2}}$ is the radius of hypercylinder, which when time changing within $-t_{G}<t<t_{G}$ first increases $0<\mathrm{R}<\mathrm{c} t_{G}$, and then decreases $\mathrm{c} t_{G}>\mathrm{R}>0$. At $\mathrm{t}=t_{G}$ we have a singularity. The radius $\mathrm{R}=t_{G}$ corresponds to the horizon in the Schwarzschild metric.

Note that this solution has an analogue in the R-region, which are considered by Denisova at al (1999). Their metrics can be rewritten as

$$
d s^{2}=c^{2} d t^{2}-d R^{2}-\left(R^{2}-R_{0}^{2}\right) d \sigma^{2} .
$$

Where $R_{0}{ }^{2}<R^{2}<\infty$. When $R^{2}=R_{0}{ }^{2}$ this metric has a naked singularity. This metric corresponds to a scalar field coupled gravitational interaction

$$
\psi=\frac{c^{2}}{2 \sqrt{k}} \ln \frac{R-R_{0}}{R+R_{0}},
$$

and physically is not connected with the interior of a black hole with collapsing scalar field, because it is another solution.

Note that after replacing the time coordinate

$$
T=\sqrt{t_{G}{ }^{2}-t^{2}}, \quad 0<T<t_{G},
$$

the obtained metric inside a $\mathrm{BH}$ with a scalar field takes the form

$$
d s^{2}=\frac{c^{2} d T^{2}}{t_{G}{ }^{2} / T-1}-d r^{2}-c^{2} T^{2} d \sigma^{2} .
$$

It turns out that scalar curvature $R_{\beta \alpha \gamma}^{\alpha} g^{\beta \gamma}$ and Krechman invariant $K=R_{\alpha \beta \alpha \gamma} R^{\alpha \beta \alpha \gamma} \quad$ on the boundary $t=0$ $\left(T=t_{G}\right)$ are the finite quantities, while in the center, when $t=t_{G}(T=0)$ they go to infinity. Since the boundary $t=0 \quad\left(T=t_{G}\right)$ is regular, therefore it can be matched with the external vacuum solution for the BH, i.e. with the Schwarzschild metric. From the matched condition of the angular parts of the metrics, we obtain the relation between the scalar charge $G$, collapsed scalar field, and the $\mathrm{BH}$ mass $M$ :

$$
G=2 M \sqrt{k} .
$$

Note that the mass function

$$
M=\frac{c^{2}}{2 k} R\left(1+(\nabla R)^{2}\right),
$$

for the given solution takes the form

$$
M=\frac{c^{2}}{2 k} \frac{c^{2} t_{G}{ }^{2}}{c \sqrt{t_{G}{ }^{2}-t^{2}}} .
$$

Hence, at the boundary value of time $t=0$, we have

$$
M(0)=\frac{c^{2}}{2 k} c t_{G}=\frac{G}{2 \sqrt{k}} .
$$

This again confirms the connection the $\mathrm{BH}$ mass with the scalar charge into the $\mathrm{BH}$. In the center of $t=t_{\mathrm{G}}$ and mass function diverge.

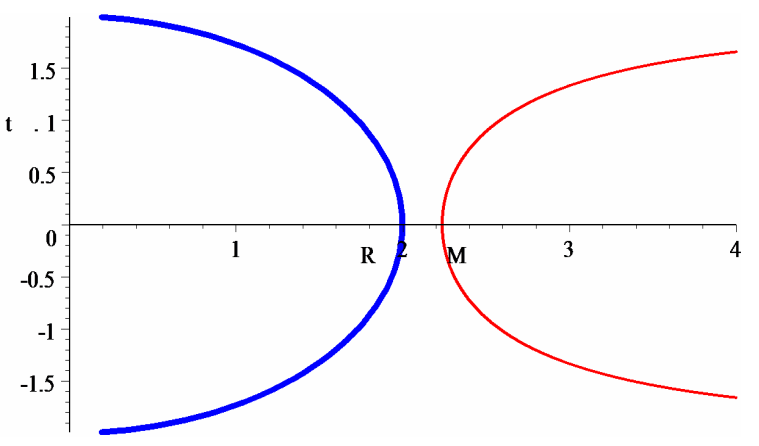

Figure 1: The behavior of the mass function and the hypercylinder radius in universe with homogeneous scalar field.

The behavior of the mass function and the hypercylinder radius represented on the figure 1 .

Here, the ordinate represents time $t$, and abscissa is the radius of hypercylinder $R$ and the mass function $M$. We see that when the time changes from $-t_{G}$ to $t_{G}=\sqrt{k} G / c^{3}=2 k M / c^{3}$ the hypercylinder radius grows from zero, reaches the maximum is equal to $c t_{G}=\sqrt{k} G / c^{2}=2 \mathrm{kM} / c^{2}$ at $t=0$ and then decreases again to zero. At the same time the mass function simultaneously decreases and reaches a minimum is equal of the mass $M(0)=c^{3} / 2 k t_{G}=G / 2 \sqrt{k}=M_{B H}$ external $\mathrm{BH}$ at $t=0$ is and rises again to infinity.

Therefore, we obtained formulas describing the evolution of the homogeneous scalar field inside the $\mathrm{BH}$. It corresponds to the final stage of the gravitational collapse some scalar field with such initial conditions that lead to the homogeneous distribution of the scalar field inside the $\mathrm{BH}$.

\section{References}

Fisher I.Z.: 1948, JETF, 18, 636.

Janis A.I, Newman E.T., Winicour J.: 1968, Phys. Rev. Lett. $20,878$.

Wyman M.: 1981, Phys. Rev. D, 24, 839.

Agnese A.G., La Camera M.: 1985, Phys. Rev. D, 31, 1280 .

Bronnikov K.A.: 1972, Preprint ITF-72-20P.

Zaitsev N.A., Kolesnikov S.M., Radynov A.G.: 1972, Preprint ITF-72-21P.

Korkina M.P.: 1976, Preprint ITF-76-93P.

Roberts M.D.: 1989, GRG, 21, 907.

Brady P.R.: 1994, Class. Quant. Grav., 11, 1255.

Oshiro Y., Nakamura K., Tomimatsu A.: 1994, Prog. Theor. Phys. (arXiv: gr-qc/9402017).

Christodoulou D.: 1987, Commun. Math. Phys. 109, 613.

Choptuik M.W.: 1993, Phys. Rev. Letters 70, 9.

Novikov I.D, Frolov V.P.: 2001, UFN, 171, 307.

Chase J.E.: 1970, Commun. Math. Phys., 19, 276.

Bekenstein J.D.: 1972, Phys. Rev. Letters, 28, 452; 1972, Phys. Rev. D, 5, 1239 and 2403.

Denisova I.P., Mehta B.V., Zubrilo A.A.: 1999, GRG, 31, 821. 